CWP-129P

DOE/ER/14079-29

November 1993

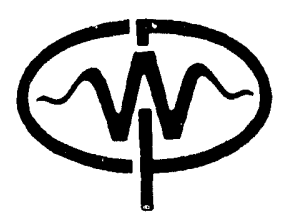

\title{
Efficient Modeling in Transversely Isotropic Inhomogeneous Media
}

\author{
Tariq Alkhalifah
}

\begin{abstract}
DISCLAIMER
This report was prepared as an account of work sponsored by an agency of the United States Government. Neither the United States Government nor any agency thereof, nor any of their employees, makes any wdrranty, express or implied, or assumes any legal liability or responsibility for the accuracy, completeness, or usefulness of any information, apparatus, product, or process disclosed, or represents that its use would not infringe privately owned rights. Reference herein to any specific commercial product, process, or service by trade name, trademark, manufacturer, or otherwise does not necessarily constitute or imply its endorsement, recommendation, or favoring by the United States Government or any agency thereof. The views and opinions of authors expressed herein do not necessarily state or reflect those of the United States Government or any agency thereof.
\end{abstract}

\footnotetext{
Center for Wave Phenomena

Colorado School of Mines

Golden, Colorado 80401

303/273-3557
} 


\title{
Efficient modeling in transversely isotropic inhomogeneous media
}

\author{
Tariq Alkhalifah
}

\begin{abstract}
An efficient modeling technique for transversely isotropic, inhomogeneous media, is developed here using a mix of analytical equations and numerical calculations. The analytic equation for the raypath in a factorized transversely isotropic (FTI) media with linear velocity variation, derived by Shearer and Chapman, is used to trace between two points. In addition, I derive an analytical equation for geometrical spreading in FTI media that aids in preserving program efficiency; however, the traveltime is calculated numerically. I then generalize the method to treat general transversely isotropic (TI) media that are not factorized anisotropic inhomogeneous by perturbing the FTI traveltimes, following the perturbation ideas of Cervený and Filho. A Kirchhoff-summation-based program relying on Trorey's (1970) diffraction method is used to generate synthetic seismograms for such a medium. For the type of velocity models treated, the program is much more efficient than finite-difference and general ray-trace modeling techniques.
\end{abstract}

\section{INTRODUCTION}

Seismic modeling programs are useful for testing the performance of algorithms for various processing needs such as dip-moveout and migration. For such tests Gray, 
Jacewitz and Epton (1991) showed the usefulness of considering simple velocity models such as those with linear volocity variation. For generating zero-offset syuthetic seismograms, ray-tracing approaches generally offer substantially better performance than do finite-difference methods when the model is assumed to have simple velocity structure, such as constant velocity or constant volocity gradient. Hale (1991) constructed a particularly efficicnt Kirchhoff-summation-based approach that exploited analytic expressions for raypaths and traveltimes in isotropic media with constant velocity gradient. His approach used the method of Trorey (1970) for reflector segmentation.

For modeling data from generally inhomogeneous, transversely isotropic (TI) media, ray tracing is computationally costly, so, again, some simplification of the velocity structure is needed to bring about desired computational efficiency. Although the tidy analytic expressions that arise for isotropic media with constant velocity gradient are no longer available for TI media, Hale's isotropic algorithm remains an excellent starting point for extending to efficient synthetic-seismogram computation for inhomogeneous TI media.

I first establish a ray-tracing algorithm for computing time and amplitude in a factorized TI (FTI) medium (i.e.. TI medium in which all ratios among the elastic coefficients for TI media are independent of position). A constant-velocity-gradient restriction is essential in establishing analytic solutions for raypaths and amplitude. Then, I use the time-perturbation equations developed by Cervený and Filho (1991) to extend from FTI to general. inhomogeneous TI media with tilted symmetry axis. Although the approach can be used to simulate offset data, here I show sample synthetic zero-offset sections only and assess the efficiency of the procedure.

Figure 1 shows a sample zero-offset synthetic seismogram for a symmetric synclinal reflector in a transversely isotropic, inhomogencous medium. The velocity is $\prime(x, z)=1.0+0.1 x+0.0 z \mathrm{~km} / \mathrm{s}$, where $x$ is distance $(\mathrm{km})$ along the surface and $z$ 
is depth measured from the surface. The asymmetry in the reflection is due to the lateral velocity variation. This data set was generated in $8.3 \mathrm{~s}$ CPU time using the IBM RS/6000 Model 520 workstation.

\section{UPGRADING SUSYNLV}

SUSYNLV - SYNthetic scismogram for Linear Velocity model- is a public domain program, authored by Hale (1991) in the Seismic Unix (SU) software system, that generates synthetic seismograms for a linear velocity function in an isotropic medium. I use the ray-tracing equations derived below to modify Hale's program to generate synthetic seismograms for constant-gradient TI media.

The modification includes the addition of a subroutine that calculates traveltimes, ray angles, and geometrical spreading for a linear velocity function in an FTI medium. In a second modification, I use a time-perturbation equation to generalize the FTI application to work for general TI media. The modified program thus now has options for both isotropic and TI media.

Computation of seismograms in this program is based on Trorey's method, in which every segment along a reflector acts as a secondary source of scattered waves. The net result of the superposition of the arrivals from linear reflecting segments along an interface is the seismogram synthesized at each geophone location, as illustrated in Figure 2. Details of the program are not important here; of importance is the fact that it requires much two-point ray tracing. In a transversely isotropic medium, where the calculation of reflection angles is relatively complicated (i.e., it requires iteration), Kirchhoff summation such as is used here is a useful tool that obviates the need for explicit computation of reflection angles.

The program as developed by Hale generates synthetic seismograms for a smoothly or linearly interpolated reflector shape beneath a homogeneous or constant-gradient medium. A model can include as many reflectors as desired; however, above each 
reflector the medium is assumed to have the same constant velocity gradient. The program also assumes muity reffection coefficient at all angles of incidence. The data may be nonzero-offset. and options exist for various source definition (e.g., exploding reflectors). These features remain in the modified program.

\section{RAY-TRACING EQUATIONS}

We shall consider only 2-D wave propagation; that is, raypaths are confined to the vertical plane containing the axis of symmetry and the velocity gradient vector. The ray tracing initially is for a P-waves traveling in FTI media with linear velocity variation in space. With these assumptions, raypaths and amplitudes can be obtained by analytic solution.

\section{Raypath and traveltime}

The eigenvalues that describe the raypath (eikonal equation) and the eigenvectors that describe the amplitudes of the various waves in inhomogeneous media are based on a high-frequency approximation. Cerveny(1972) shows that the eigenvalues are solutions of

$\operatorname{Det}\left(\Gamma_{j k}-G_{m} \delta_{j k}\right)=0$

where $\Gamma_{j k}$ are components of a $3 \times 3$ matrix $\Gamma$ given by the relation

$\Gamma_{i k}\left(x_{s}, p_{s}\right)=a_{i j k l}\left(x_{s}\right) p_{j} p_{l}$. (smmmation convention for repeated subscripts)

$p_{s}=\partial \tau / \partial x_{s}, a_{i j k l}=c_{i j k l} / \rho$,

where $\tau$ is traveltime along the ray, $\rho$ is density, $x_{s}$ are position coordinates $(s=1,2,3)$, 
and $c_{i j \ltimes i}$ are the elastic coefficients, in general, functions of $x_{s}$.

The $G_{m}$ are the three eigenvalues repiesenting the eikonal equation for the three wave t?pes: quasi P-wave when $m=1$; quasi SV-wave when $m=2$; and quasi $S H$-wave when $m=3$. For P-waves (I shall drop the "quasi" modifier in the following),

$$
G_{1}\left(x_{s}, p_{s}\right)=1
$$

For example, for isot ropic media, $G_{1}=\alpha^{2}\left(x_{s}\right) p_{i} p_{i}$, where $\alpha\left(x_{s}\right)$ is the P-wave velocity.

In a factorized anisotropic inhomogeneous (FAI) medium

$$
a_{i j k l}\left(x_{s}\right)=f^{2}\left(x_{s}\right) A_{i j k l}
$$

where $A_{i j k l} \equiv \frac{a_{, j k}\left(r_{s}\right)}{a_{3333}\left(x_{s}\right)}$ are position-independent ratios of the density-normalized elastic coefficient, and $f\left(x_{s}\right)=\left[a_{3333}\left(x_{s}\right)\right]^{\frac{1}{2}}$ is the velocity in the direction parallel to the symmetry axis at position $x_{s}$. Equation (1) then becomes

$$
G_{1}\left(x_{s}, p_{s}\right)=f^{2}\left(x_{s}\right) G_{1}^{o}\left(p_{s}\right)=1
$$

where $G_{1}^{o}\left(p_{i}\right)$ is P-wave eigenvalue expression (1) with the $a_{i j k l}\left(x_{s}\right)$ coefficients replaced by the ratios $A_{i j k l}$.

For a constant velocity gradient,

$$
f\left(x_{s}\right)=A_{s} x_{s}
$$

where $t_{s}$ is the gradient in the $x_{s}$ direction. For such inhomogeneity, Shearer and Chapman (1988) showed the following simple relation between the slowness vector and the position vector.

$$
p_{i} x_{i}=0
$$

For 2-D, substituting equations (3) and (4) into equation (2) results in the raypath equation for a generally anisotropic medium with constant velocity gradients in the $x_{1}$ and $x_{3}$ directions.

$$
\left(A_{3} p_{1}-A_{1} p_{3}\right)^{2} G_{1}^{o}\left(x_{3},-x_{1}\right)=1
$$


Note that, for media of the trpe considered here, the lactor $A_{3} p_{1}-A_{1} p_{3}$ is independent of position along a ray:

For the special case of a transversely isotropic medium, the position-independent eigenvalue for the P-wave is given by (White, 1083)

$$
G_{1}^{o}\left(p_{1}, p_{3}\right)=0.5\left\{(a+l) p_{1}^{2}+(c+l) p_{3}^{2}+\left\{\left[(a-l) p_{1}^{2}-(c-l) p_{3}^{2}\right]^{2}+4(f+l)^{2} p_{1}^{2} p_{3}^{2}\right\}^{\frac{1}{2}}\right\}
$$

where $a \equiv A_{1111}, c \equiv A_{33333}, f \equiv A_{1133}$, and $l \equiv A_{1313}$.

For axis of symmetry in the $x_{3}$ (vertical) direction, equation (6), along with equation (4), gives (Larner. 1993)

$$
G_{1}^{o}\left(x_{3},-x_{1}\right)=0.5\left\{(a+l) \cdot x_{3}^{2}+(c+l) \cdot x_{1}^{2}+\left\{\left[(a-l) \cdot x_{3}^{2}-(c-l) x_{1}^{2}\right]^{2}+4(f+l)^{2} x_{3}^{2} x_{1}^{2}\right\}^{\frac{1}{2}}\right\} .
$$

Now, equation $(t)$ gives

$$
p_{3}\left(p_{1}\right)=-\frac{x_{1}}{x_{3}} p_{1},
$$

which, for later purposes. I use in defining the distance

$$
r_{0}=r_{0}\left[p_{1}\left(\xi_{0}\right)\right] \equiv \frac{1}{A_{3} p_{1}\left(\xi_{0}\right)-A_{1} p_{3}\left[p_{1}\left(\xi_{0}\right)\right]}
$$

where $\xi$ is a rumning parameter that monotonically increases along the ray, with $\xi=\xi_{0}$ at the starting point of the ray (see Figure 3). Specifically,

$$
d \xi=\frac{1}{f\left(x_{i}\right)} d \tau
$$

The value of the ray parameter $p_{1}\left(\xi_{0}\right)$ at $\xi_{0}$ defines the particular raypath. Then, distance $r_{0}$, as seen in equation (8), is defined in terms of $p_{1}\left(\xi_{0}\right)$, as shown in Figure 3.

In an isotropic medium with constant velocity gradient, all raypaths are circles. Consider an isotropic medium with velocity $f\left(x_{i}\right)$ identical to the vertical velocity in the constant-gradient FTI medium. Then, $r_{0}$, as defined in equation (8), is the radius of the circular raypath whose center is at the origin $\left(x_{1}=0, x_{3}=0\right)$ and 
goes through the point $b$, in Figure 3. that is associated with the raypath for the TI medium. Substituting equation (8) into equation (5) gives

$$
G_{1}^{o}\left(x_{3},-x_{1}\right)=r_{0}^{2}
$$

which, along with ( 7 ), results in the quadratic raypath equation for the TI medium

$$
a x_{1}^{1}+3\left(r_{0}, x_{3}^{2}\right) x_{1}^{2}+\gamma\left(r_{0}, x_{3}^{2}\right)=0
$$

for $x_{1}$ as a function of $x_{3}$ and $r_{11}$. Here, the coefficient $\alpha$ is a function of only the elasticity coefficients, and the coefficients $\beta$ and $\gamma$ have the additional dependencies shown.

Follown"g Cervený (1989), for constant velocity gradient [equation (3)],

$$
\begin{aligned}
f\left(x_{1}, x_{3}\right) & =A_{1} x_{1}+A_{3} x_{3}, \\
p_{1}(\xi) & =p_{1}\left(\xi_{0}\right)-A_{1}\left(\xi-\xi_{0}\right), \\
p_{3}(\xi) & =p_{3}\left(\xi_{0}\right)-A_{3}\left(\xi-\xi_{0}\right) .
\end{aligned}
$$

Then, traveltime along the ray is given by

$$
\tau(\xi)=\tau\left(\xi_{0}\right)+\int_{\xi_{0}}^{\xi}\left[G^{o}\left(p_{1}, p_{3}\right)\right]^{-\frac{1}{2}} d \xi .
$$

\section{Geometric spreading}

Although geometric spreading in an isotropic medium with constant velocity gradient can be expressed analytically, the situation is not that simple for FTI media, which require dealing with the distinction between group and phase velocities. I use a scaling technique to get an approximate expression for the geometric spreading in such a medium.

First, consider the geometric spreading function $q$, a quantity proportional to the distance $l$ between the central ray and a nearby paraxial ray. From Cervený (1981) 
it follows that

$$
q=k \cdot l=\int_{s_{0}}^{s} l(s) d s .
$$

where $u(s)$ is the volocitr. s is the arelength along a computed ray - the central ray, and $k$ is a proportionality constant. A paraxial ray is a ray (not actually traced) in the vicinity of the central ray, its normal distance from the central ray describing the geometrical spreading (Corveny, 1981) along the central ray. Figure 4 shows a central and paraxial ray for a transversely isotropic medium, and Figure 5 shows the two rays for its isotropic counterpart. Note, in Figure 5, that although the paraxial ray, like the central ray, is circular, the distance $r_{p}$ from the center of the central ray to the paraxial ray depends on the position along the central ray.

In an isotropic. constant-gradient medium, Appendix A shows that equation (9) becomes

$$
q=r_{0} x \sqrt{A_{1}^{2}+A_{3}^{2}}
$$

where $r_{0}=\left(x_{1}^{2}+x_{3}^{2}\right)^{\frac{1}{2}}$ is the same $r_{0}$ given in equation (8), and $x$ is the distance, measured along the normal to the gradient direction, between the ray takeoff point and the point along the ray where $q$ is measured. We now seek a comparably simple, approximate expression for the geometric spreading function $q_{t}$ for FTI media.

The keys to estimating $q_{t}$ are scaling relationships, the first of which is the underlying similarity between the ray equation (j) and the slowness equation (2) for FTI media. This similarity is shown graphically in a comparison of the ravpaths shown in Figure 3 and the slowness curves in Figure 6. Shearer and Chapman (1988) have shown that the raypaths for both the FTI and isotropic media are scaled, 90-degree rotated versions of the respective slowness curves. Now, define the distance from the origin to a point $\xi$ along the raypath as $r_{t}(\theta) \equiv \sqrt{x_{1}^{2}[\xi(\theta)]+x_{3}^{2}[\xi(\theta)]}$, where $\theta$ is the angle between the line connecting these two points and the horizontal axis, as shown in Figure 3. Also, as shown in Figure 6, define the slowness of the ray at that same point $\xi$ as $p(\theta) \equiv \sqrt{p_{1}^{2}[\xi(\theta)]+p_{3}^{2}[\xi(\theta)]}$, where the angle $\theta$ is now seen to be the angle of 
the slowness vector with the vertical slowness axis (axis of symmetry, here). Consider $r_{0}=x_{1}(\xi)$ where $x_{3}(\xi)=0$, and $p_{0}=p_{3}(\xi)$ where $p_{1}(\xi)=0$, as reference values for position and slowness, respectively. From the similarity of the FTI curves in Figures 1 and 4 ,

$$
r_{t}(\theta)=\frac{p(\theta)}{p_{0}} r_{0} .
$$

But $r_{0}$ and $p_{0}$ are just the radii of the circular raypath and slowness curve for the isotropic counterpart of the FTI medium, shown in Figures 1 and 4. Therefore, equation (11) is a scaling relationship between the distance $r_{t}(\theta)$ and $r_{0}$ for the two media.

Exploiting this scaing relationship, I can establish an equation for the geometrical spreading function $q_{t}$ in FTI media. The scaling relationship given by equation (11) pertains to the central ray. For the paraxial rays this relationship is only approximate because I am using the origin for the central rays in describing the paraxial rays. I assert that the following approximate relationship holds for paraxial rays of the TI and isotropic media.

$$
r_{t p}(\theta) \doteq \frac{p(\theta)}{p_{0}} r_{p}(\theta),
$$

where $r_{p}$ is the distance from the origin to the paraxial ray for the isotropic medium, and $r_{t p}$ is the distance from the origin to the paraxial ray for the FTI medium. This approximate relationship holds because the difference in takeuff angle for the central ray and the paraxial ray is small (see Figures 2 and 3). From equation (9),

$$
q_{t}=k \cdot l_{t}
$$

where $l_{t}$ is the distance between the central ray and a paraxial ray for the FTI medium measured along the normal to the phase direction (wavefront direction). But, from Figure 7

$$
l_{t}(\theta)=\left[r_{t p}(\theta)-r_{t}(\theta)\right] .
$$


Inserting equation (14) into equation (13) and using equations (11) and (12), we get

$$
q_{t}=k \cdot\left[r_{p}(\theta)-r_{0}\right] \frac{p(\theta)}{p_{0}}
$$

But for the isotropic medium

$$
q=k l=k\left[r_{p}(\theta)-r_{0}\right]
$$

so equation (10) becomes

$$
q_{t}=\frac{p(\theta)}{p_{0}} q=\frac{r_{t}(\theta)}{r_{0}} q
$$

The second equality here follows from equation (11).

Substituting equation (10) into equation (16) gives the geometric spreading function in the FTI medium,

$$
q_{t}=r_{t} x \sqrt{A_{1}^{2}+A_{3}^{2}}
$$

\section{Ray tracing between two points}

I now use the scaling technique once again, this time to iteratively find the angle $\theta_{0}$ for the ray originating at source position $\left[x_{1}\left(\xi_{0}\right), x_{3}\left(\xi_{0}\right)\right]$ and ending at diffractor position $\left[x_{1}\left(\xi_{1}\right), x_{3}\left(\xi_{1}\right)\right]$ in an FTI medium with general velocity-gradient direction.

The non-vertical, constant-velocity gradient has magnitude

$$
g=\sqrt{A_{1}^{2}+A_{3}^{2}}
$$

and angle from vertical

$$
\nu=\tan ^{-1} \frac{A_{1}}{A_{3}}
$$

As shown in Figure 8, the distance from the origin to the reference position (ray starting point), measured along the gradient direction, is

$$
z_{g}=\frac{v_{0}}{g}
$$


where $v_{0}$ is the velocity in the vertical direction at the starting point. I use

$$
\cos \varphi=\frac{A_{3}}{\sqrt{A_{1}^{2}+A_{3}^{2}}}
$$

and

$$
\sin \varphi=\frac{A_{1}}{\sqrt{A_{1}^{2}+A_{3}^{2}}}
$$

to rotate the coordinates $x_{1}$ and $x_{3}$ to new coordinates $x_{1}^{T}$ and $x_{3}^{T}$, where $x_{3}^{T}$ is along the gradient-vector direction. Consider the distance in the $x_{3}^{T}$ direction between position $\left[x_{1}\left(\xi_{0}\right), x_{3}\left(\xi_{0}\right)\right]$ and position $\left[x_{1}\left(\xi_{1}\right), x_{3}\left(\xi_{1}\right)\right]$,

$$
\Delta x_{3}^{T} \equiv x_{3}^{T}\left(\xi_{1}\right)-x_{3}^{T}\left(\xi_{0}\right),
$$

which I use to define

$$
z \equiv \Delta x_{3}^{T}+z_{g},
$$

as the distance in the $x_{3}^{T}$ direction from the origin to position $\left[x_{1}\left(\xi_{1}\right), x_{3}\left(\xi_{1}\right)\right]$.

Using scaling relationship (11) once for angle $\theta_{0}$ at $\xi_{0}$ and again for angle $\theta_{1}$ at $\xi_{1}$ along the ray (see Figure 8), gives

$$
r_{t}\left(\theta_{1}\right)=\frac{p\left(\theta_{1}\right)}{p\left(\theta_{0}\right)} r_{t}\left(\theta_{0}\right) .
$$

From Figure 8 .

$$
r_{t}\left(\theta_{1}\right)=\frac{z}{\sin \psi_{1}}
$$

and

$$
r_{t}\left(\theta_{0}\right)=\frac{z_{g}}{\sin \psi_{0}},
$$

where $\psi_{0}$ and $\psi_{1}$ are angles from the $x_{1}^{T}$ axis to lines connecting the origin with $\xi_{0}$ and $\xi_{1}$, respectively. Also from Figure 8 ,

$$
\psi_{0}=\theta_{0}-\varphi
$$

and

$$
\psi_{1}=\theta_{1}-\varphi .
$$


Substituting equations (18) and (19) into equation (17) gives

$$
\frac{z}{\sin \psi_{1}}=\frac{p\left(\psi_{1}+\varphi\right)}{p\left(\psi_{0}+\varphi\right)} \frac{z_{y j}}{\sin \psi_{0}},
$$

with two unknowns. $\iota_{1}$ and $\iota_{0}$. The known value of $\Delta x_{1}^{T}$ satisfies

$$
\Delta x_{1}^{T} \equiv x_{1}^{T}\left(\xi_{1}\right)-x_{1}^{T}\left(\xi_{0}\right)=\frac{x_{3}^{T}\left(\xi_{1}\right)}{\tan \psi_{1}}-\frac{x_{3}^{T}\left(\xi_{0}\right)}{\tan \psi_{0}},
$$

which gives

$$
\psi_{1}\left(\psi_{0}\right)=\tan ^{-1} \frac{x_{3}^{T}\left(\xi_{1}\right) \tan \psi_{0}}{x_{3}^{T}\left(\xi_{0}\right)+\Delta x_{1}^{T} \tan \psi_{0}} .
$$

Combining equations (20) and (21) yields a single transcendental equation for $\psi_{0}$, the takeoff phase-velocity angle from $x_{1}^{T}$ for the two-point ray-tracing problem. A root-finding algorithm (e.g.. the Newton-Raphson method or the secant method) is used to solve for $\psi_{0}$. Note from Figure 8 that if $\Delta x_{1}^{T} \tan \psi_{0}$ is bigger than $x_{3}^{T}$, then the diffractor position is past the turning point of the ray.

The scaling approach is an alternative to the general raypath equation for twopoint ray tracing. It gives a simpler way of treating rotation in the gradient direction; rotation of the ravpath equation is relatively complicated. Furthermore, the scaling approach helps to recognize whether or not the diffraction position is beyond the turning point of the ray, a necessary step in evaluating the sign of the ray parameters in equation ( 4$)$.

For arbitrary orientation of symmetry axis, a rotation of the coordinate axes is done in the program such that $x_{3}$ is always in the clirection of the axis of symmetry for the FTI medium. This rotation is simpler than the rotation used in the two-point ray-tracing because it involves a rotation of the whole problem.

\section{From FTI to general anisotropy}

While still making use of efficiency of the analytic solutions, the anisotropy condition can be generalized through a perturbation of the traveltimes and geometric spreading for the FTI medium. 
Consider two media: (1) a background medium (unperturbed), possibly anisotropic and inhomogeneous, in which we do our general ray tracing, and (2) a perturbed medium. which may also be anisotropic and inhomogeneous, in which we wish to calculate traveltime and amplitude. The traveltime in the perturbed medium $T(a, b)$ along a ray traveling from point $a$ to point $b$ is calculated from the traveltime in the background medium $T^{0}(a, b)$ traveling between the same two points. I seek an efficient scheme for computing the time perturbation $\delta T(a, b) \equiv T(a, b)-T^{0}(a, b)$, given $T^{0}(a, b)$. Differences between the density-normalized elastic parameters in the background medium $a_{i j k l}^{0}$ and those in the perturbed medium $a_{i j k l}$ define the degree of perturbation $\delta a_{i j k l}$ as follows

$$
\delta a_{i j k l}=a_{i j k l}-a_{i j k l}^{0}
$$

Following Červený and Filho (1991), for a generaliy anisotropic inhomogeneous background,

$$
\delta T_{P}(a, b)=-\frac{1}{2} \int_{a}^{b} \delta a_{i j k l} p_{i} p_{l} m_{j} m_{k} d T
$$

where $m_{i}$ are the components of the eigenvector $\mathbf{m}$ (the direction of the particle motion). here for the $\mathrm{P}$-wave. The integration in equation (22) is performed along a ray $L_{0}$ computed in the background medium, and quantities $p_{i}, p_{l}, m_{j}, m_{k}$ and $d T$ pertain to the background medium.

Take the integrand in equation (22) to define the anisotropy perturbation factor $F$ for the homogeneous, isotropic segment,

$$
F \equiv-\frac{1}{2} \delta a_{i j k l} p_{i} p_{l} m_{j} m_{k}
$$

Now, consider the geometric spreading function $q$ along the ray in the background medium (e.g., in a homogeneous isotropic medium, $q=\alpha^{2} T^{o}$ ). Then, the first-order perturbation of the geometrical spreading for the ravpath between point $a$ and point 
$b$ (Alkhalifah, 1993), is given by

$$
\lambda q(a, b)=\int_{a}^{b}-F d q,
$$

where $F$ and $d q$ pertain to the background medium. and $F \ll 1$.

\section{SYNTHETIC DATA EXAMPLES}

I will use a single TI medium to show synthetic lata generated by this modelling program. The Wills Point shale with Thomsen's anisotropy parameters $\delta=0.315$ and $\epsilon=0.215$ (Thomsen, 1986) is considered to be a relatively strongly anisotropic medium. Positive angle between the symmetry axis and the vertical, $\psi$. corresponds to symmetry axis rotated clockwise from the vertical, and negative $\psi$ is just the opposite.

Figure 9 shows synthetic seismograms resulting from modeling two point scatters located at midpoint $2.5 \mathrm{~km}$, and depths 1 and $3 \mathrm{~km}$. The vertical velocities for all the models $v(z)=2.0+0.6 z \mathrm{~km} / \mathrm{s}$. Figure 9a shows the diffraction curves for an isotropic medium; Figure $9 \mathrm{~b}$ for a Wills Point shale FTI medium with a vertical axis of symmetry $(\psi=0)$; Figure $9 \mathrm{c}$ for the same FTI medium with $\psi=30$ degrees; and Figure 9d for the same FTI medium with lateral as well as vertical velocity variation. $v(x, z)=2.0+0.1 x+0.6 z \mathrm{~km} / \mathrm{s}$, and with $\psi=-40$ degrees. Note that for Figures $9 \mathrm{a}$ and $9 \mathrm{~b}$ the diffraction curves, as expected, are symmetric; however, in Figures $9 \mathrm{c}$ and $9 \mathrm{~d}$ they are asymmetric and laterally shifted. In Figure $9 \mathrm{c}$, the asymmetry and shift are due solely to the tilt of the symmetry axis. In Figure 9d, both the lateral velocity variations and the nonvertical symmetry axis contribute (oppositely, here) to the asymmetry and shift.

The lateral shift in Figure 9c implies migration that ignores the tilted symmetry axis, such as conventional isotropic migration, will yield lateral mispositioning of reflectors, even horizontal ones, just as happens in media with laterally varying 
overburden, even though the medium here has no lateral variation.

Figure 10 shows an undulating-reflector model, and Figure 11 shows synthetic seismograms corresponding to that model. The vertical velocity this time is held at $v(z)=3.0+0.5 z \mathrm{~km} / \mathrm{s}$. Figure $11 \mathrm{a}$ corresponds to an isotropic medium; Figure $11 \mathrm{~b}$ to the Wills Point shale FTI medium with $\psi=0$ degrees; Figure 11c to the same FTI medium with $\psi=-30$ degrees; and 11d to the same FTI medium with lateral and vertical velocity variation, $v(x, z)=3.0+0.5 x+0.5 z \mathrm{~km} / \mathrm{s}$, with $\psi=-40$ degrees. The asymmetry and shift, mentioned above, is observed here, as well. The reflector shown in Figure 10 is just a summation of scattering points, and the reflector response in Figure 11 is a superposition of diffraction curves such as those in Figure 9.

Figure 12 shows more synthetic seismograms generated for the reflector model in Figure 10. This time, the velocity structure $v(z)=4.0-0.1 z \mathrm{~km} / \mathrm{s}$, corresponding to a decrease in the vertical velocity with depth, a situation not generally encountered in practice. Figure 12a corresponds to the Wills Point shale with $\psi=0$ degrees, and Figure $12 \mathrm{~b}$ with $\psi=-30$ degrees. Clearly, the program is not restricted to modeling velocities that only increase with depth.

Next, I lift the restriction that the medium be factorized anisotropic. Figure 13 shows synthetic seismograms for (a) the same two diffractors used in generating Figure 9, and (b) the reflector model in Figure 10, but these seismograms were generated for a TI medium using the time-perturbation feature. The time perturbation is from the FTI Wills Point shale, to another anisotropic medium, a general TI medium, in which $\delta$ and $\epsilon$ change with depth. Specifically, the perturbation includes the following changes of elastic coefficients: $\frac{d l}{d z}=0.05$ and $\frac{d a}{d z}=0.1$, where $l$ and $a$ are the coefficient ratios given in equation (6), and $\delta=0.315$ and $\epsilon=0.215$ at the surface. Comparing Figure 13a with Figures 9a and 9b, note that Figure 13a suggests more extreme anisotropy than does Figure $9 b$; that is, the diffraction curves in Figure 13a show more departure from those for the isotropic medium than do the curves in 
Figure $9 \mathrm{~b}$, despite the fact that all models have the same vertical root-mean-square velocity. I attribute this behavior to the increase in size of the anisotropy parameters with depth.

\section{EFFICIENCY}

The primary objective of constructing the original isotropic modeling program was efficiency in generating synthetic data. Similarly my goal was to preserve that essential efficiency while extending to inhomogeneous TI media. Generating synthetic data for such media by means such as finite-difference or general ray tracing is slow and costly. For ray tracing approaches. speed will be slowed down primarily by the iterative numerical integration needed to perform ray tracing between two points. The approach described here does not require such costly iteration.

The synthetic data generated for Figure 9 and Figure 13b contain 201 common midpoints at an interval of $0.05 \mathrm{~km}$. and 401 time samples with a time interval of $0.01 \mathrm{~s}$. The peak frequency used is $15 \mathrm{~Hz}$. Table 1 shows CPU times, on the IBM RS/6000 Model 520 workstation, needed to generate these synthetic seismograms. For the FTI time shown in Table 1, I chose the largest of the times for the three FTI cases in Figure 9. For comparision. a finite-difference solution for the full elastic-wave equation for transversely isotropic media with vertical symmetry axis would require on the order of $1500 \mathrm{~s}$.

\begin{tabular}{|c|c|}
\hline Technique & CPU time (s) \\
\hline \hline Isotropic (Figure 9) & 0.6 \\
\hline FTI (Figure 9) & 1.5 \\
\hline general TI (Figure 13a) & 2.1 \\
\hline
\end{tabular}

Table 1. Compute times for various two-diffractor tests.

The synthetic data generated in Figure 11 and Figure 13b contain 201 common 
midpoints at an interval of $0.05 \mathrm{~km}$. and 501 time samples at $0.01 \mathrm{~s} \mathrm{time} \mathrm{interval.}$ The peak frequency is again $15 \mathrm{~Hz}$. Table 2 shows the CPU times needed to generate those data.

\begin{tabular}{|c|c|}
\hline Technique & CPU time (s) \\
\hline \hline Isotropic (Figure 11) & 5.6 \\
\hline FTI (Figure 11) & 36.9 \\
\hline general TI (Figure 13b) & 52.0 \\
\hline
\end{tabular}

Table 2. Compute times for various tests with the reflector model in Figure 8.

The ray-trace times in Table 2 are much larger than those in Table 1 because seismogram computation for the model in Figure 10 required summation of contributions from 246 diffracting segments along the reflector, whereas only two diffracting segments were used for the tests in Figure 9 and 13a. However, the time increase is much lower than predicted by the number of reflector segments beca'se the times in Table 1 are dominated by the program overhead. For the class of models allowed in this program, the efficiency demonstrated here far exceeds that of either general ray tracing (Červený, 1989) or finite-difference techniques.

\section{CONCLUSION}

The ray-tracing procedure for FTI and general TI media should be useful in generating models for applications such as algorithm testing in $\mathrm{TI}$ inhomogeneous media, including migration and dip-moveout studies. Alkhalifah (1993), for example, used synthetic data generated by this program in tests of Gaussian beam migration for FTI media. Whereas. for FTI media with constant velocity gradient, computed raypaths are exact and traveltimes are accurately computed by numerical integration, for more general anisotropy I modify those times by the perturbation procedure so that they become only approximate. Alkhalifah (1993), however, shows that the errors 
are negligible when the perturbation is less than about 20 percent.

The key characteristic of this program is the efficiency with which it generates seismograms. This efficiency is bronght about by the analytical equation for geometrical spreading, derived here. This geometrical-spreading equation for FTI media could have other applications such as in study of amplitude-versus-offset (AVO) dependency on $\mathrm{TI}$ parametors in inhomogeneous media.

\section{ACKNOWLEDGEMENTS}

I thank Kien Larner for his critical suggestions and review of the paper. Professor Larner also provided me the idea for this study. I also thank V. Cervený for helping me understand some practical issues in ray tracing. In addition, I thank Mohammed Alfaraj for his time in introducing me to the Center for Wave Phenomena (CWP) environment. Thanks are due to the CWP, Colorado School of Mines, for its technical support, and also to KACST. Saudi Arabia, for its financial support. Financial support for this work also was provided in part by the United States Department of Energy, (this support does not constitute an endorsement by DOE of the views expressed in this paper) and by the members of the Consortium Project on Seismic Inverse Methods for Complex Structures at the Center for Wave Phenomena. Colorado School of Mines.

\section{REFERENCES}

Alkhalifah, T., 1993, Gaussian beam migration for anisotropic media: CWP Project Review, CWP-137, Colorado School of Mines.

Červený, V., 1972, Scismic rays and ray intensities in inhomogeneous anisotropic media: Geophys. J. R. astr. Soc., 29, 1-13.

Cervený, V., 1981, Computation of geometrical spreading by dynamic ray tracing: Stanford Exploration Project, 28, 49-59. 
Cervený, V., and Simoes-Filho. I. A., 1991, The traveltime perturbations for seismic

. body waves in factorized anisotropic inhomogeneous media: Geophys. J. Int., 107, 210-229.

Cervený, V., 1989, Ray tracing in factorized anisotropic inhomogeneons media: Geophys. J. Int., 94, $57 j$ j80.

Hale, D., 1991, SUSYXLY: Part of the CWP public domain software package SU (seismic programs for LNIX based machines), Colorado School of Mines.

Gray, S. H., Jacewit\%. C. A., and Epton. M. E., 1991, Analytic synthetic seismograms for depth migration testing: Geophysics, 56, 697-700.

Larner, K., 1993, Dip-moveout error in transversely isotropic media with linear velocity variation in depth: Geophysics, 58. 1442-1453.

Shearer, P. M. and Chapman, C. H., 1988, Ray tracing in anisotropic media with linear velocity gradient: Geophys.J. Int., 94, 575-580.

Thomsen. L., 1986, Weak elastic anisotropy: Geophysics, 51, 1954-1966.

Trorey, A. WV., 1970. A simple theory for seismic diffractions: Geoplysics, 35, 762784.

White, J. E., 1983, Lnderground sound: Application of sound waves: Elsevier, $253 \mathrm{p}$.

\section{APPENDIX A: GEOMETRIC SPREADING FUNCTION FOR ISOTROPIC MEDIA WITH CONSTANT VELOCITY GRADIENT}

First, consider the geometric spreading function $q$ in a $v(z)$ medium,

$$
q=\int_{s_{0}}^{s} v(s) d s
$$

where $s$ is the arclength. For circular raypaths,

$$
s=r_{0}\left(\theta-\theta_{0}\right)
$$


$\theta_{0}$ is the takeoff angle from the gradient direction and $2 \pi r_{0}$ is the circumference of the circular raypath. Then.

$$
d s=r_{0} d \theta \text {. }
$$

Substituting equation (A-2) into equation $(A-1)$ gives

$$
\eta=r_{0} \int_{\theta_{0}}^{\theta} v(s) d \theta
$$

For

$$
v(s)=v_{0}+g z(s)
$$

where $g=\sqrt{f_{1}^{2}+f_{3}^{2}}=$ is in the gradient direction, and $v_{0}$ is the velocity at the takeoff point.

$$
r(s)=r_{0}+g\left(r_{0} \sin \theta(s)-z_{g}\right),
$$

where $z_{g}=\frac{10}{g}$. giving

$$
l^{\prime}(s)=g r_{0} \sin \theta
$$

So. substituting equation $(A-4)$ into equation $(A-3)$ gives

$$
q=g r_{0}^{2} \int_{\theta_{0}}^{\theta} \sin \theta d \theta
$$

Integrating equation $(A-j)$ yiolds

$$
q=g r_{0} x
$$

where $x$ is the distance, measured along the normal to the gradient direction, between the ray takeoff point and the point along the ray, i.c., $x=r_{0}\left(\cos \theta-\cos \theta_{0}\right)$. 


\section{FIGURE CAPTIONS}

Fig 1 Synthetic seismogram of a syncline (shown in top) in a TI medium with velocity $r(x, z)=1.0+() .1, x+0.6=\mathrm{km} / \mathrm{s}$. The data consist of 101 traces, and the wavelet used is a Ricker wavelet with dominant frequency of $5 \mathrm{H}$.

Fig 2 Schematic depth depicting raypaths from a source to a number of points along a reflector, and then back to a receiver. For simplicity in the figure, the raypaths are drawn as straight lines. as for a homogeneous medium. The solid thick line represents the geometric-reflection ravpath (i.c., one that obeys Snell's law).

Fig 3 Raypatlis in a constant-gradient, transversily isotropje medium and in its isotropic counterpart. The velocity $f\left(x_{s}\right)$ in the isotropic medium is identical to the vertical velocity in the FTI medium.

Fig 4 A central ray and nearby paraxial ray for a transversely isotropic medium.

Fig 5 A central ray and nearby paraxial ray for the isotropic counterpart of the medium in Figure 3.

FIg 6 The slowness curve for an FTI medium and its isotropic counterpart.

Fig 7 The scaling relations that exists between a ray traveling in a constant-gradient FTI medium and its isotropic counterpart.

Fig 8 Parameters that correspond to the case of non-vertical volocity gradient.

Fig 9 Zero-offset syuthetic seismograms of two diffractor points for (a) an lisotropic medium, (b) Wills Point shale FTI medium with vertical axis of symmetry $(\varphi=0)$, (c) the same FTI medium with $\psi=30$ degrees, and (d) the same FTI medium with $\iota^{\prime}=-40$ degrees and velocity $\iota^{\prime}(x, z)=2.0+0.1 x+0.6 z \mathrm{~km} / \mathrm{s}$. The volocity in (a), (b) and (c) is $v(z)=2.0+0.6 z \mathrm{~km} / \mathrm{s}$. 
Fig 10 Reflector model.

Fig 11 Zero-offset synthetic seismograms corresponding the reffector moded in Figure 9 for (a) an isotropic medium. (b) Wills Point shale FTI medium with vertical axis of symmetry $(\iota=0$ degrees), (c) the same FTI medium with $\iota=30$ degrees. and (d) the sanne FTI medium with $\psi=-40$ degrees and velocity $u^{\prime}(r, z)=3.0+0.5 . r+0.5: \mathrm{km} / \mathrm{s}$. The velocity in $(\mathrm{a}),(b)$ and $(c)$ is $u(z)=3.0+0.5$ $=\mathrm{km} / \mathrm{s}$.

Fig 12 Zero-offset syiuthetic soismograms corresponding the reflector model in Figure 9 for (a) the Wills Point shale FTI mediun with vertical axis of symmetry $(\varphi=0)$, and (h) the same FTI medium with $\iota=-30$ degrees. The velocity structure here is $l^{\prime}(z)=4.0-0.1 \mathrm{z} \mathrm{km} / \mathrm{s}$.

FIG 13 Zero-offset syuthetic seismograms corresponding to (a) the two diffraction points used in Figure 8, and (b) the reflector model used in Figure 10b. Here, time perturbation is used with $\frac{d l}{d z}=0.05$ and $\frac{d a}{d z}=0.1$ for both plots. The symmetry axis is vertical. 
1 .
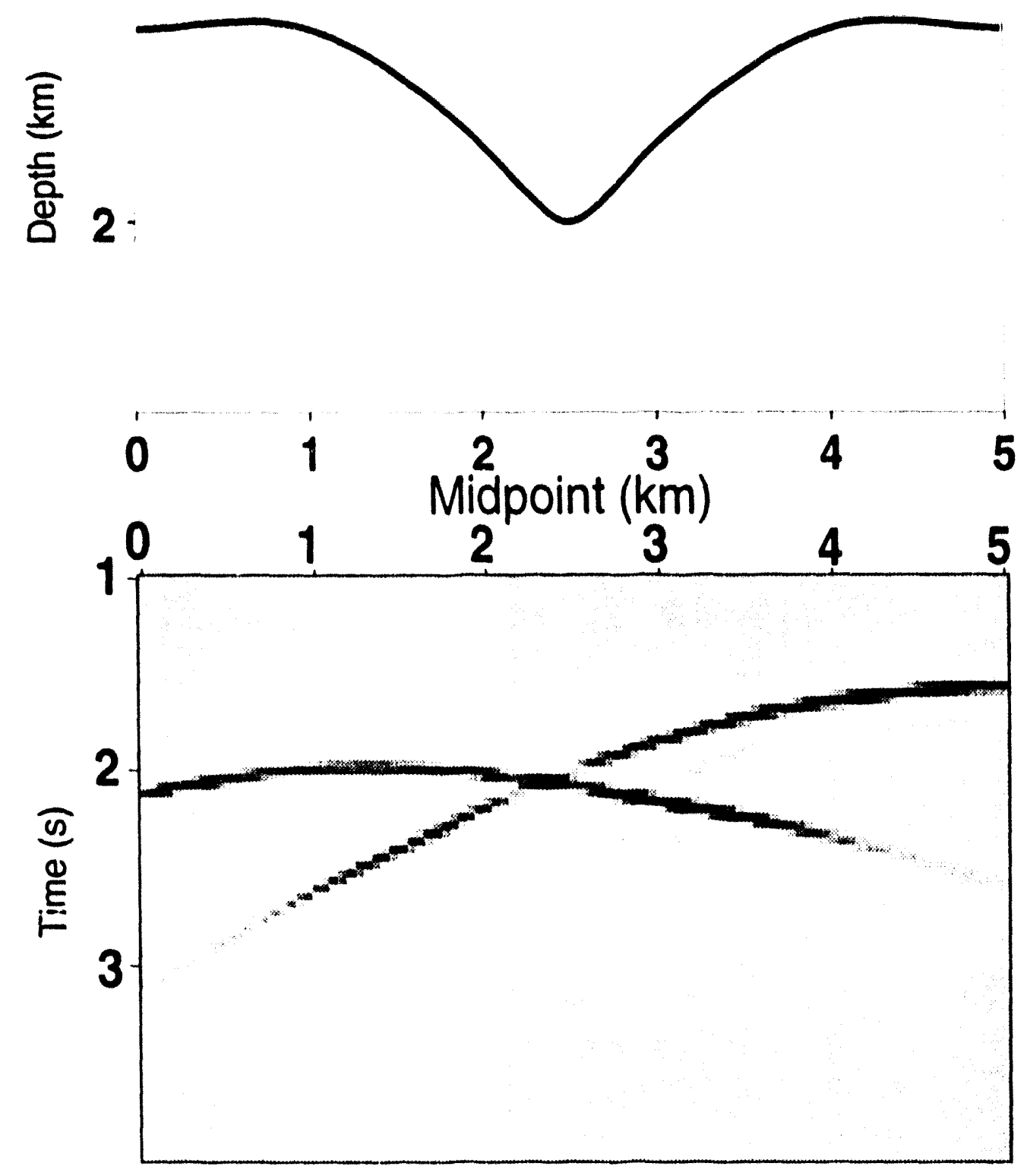

FIG. 1. 


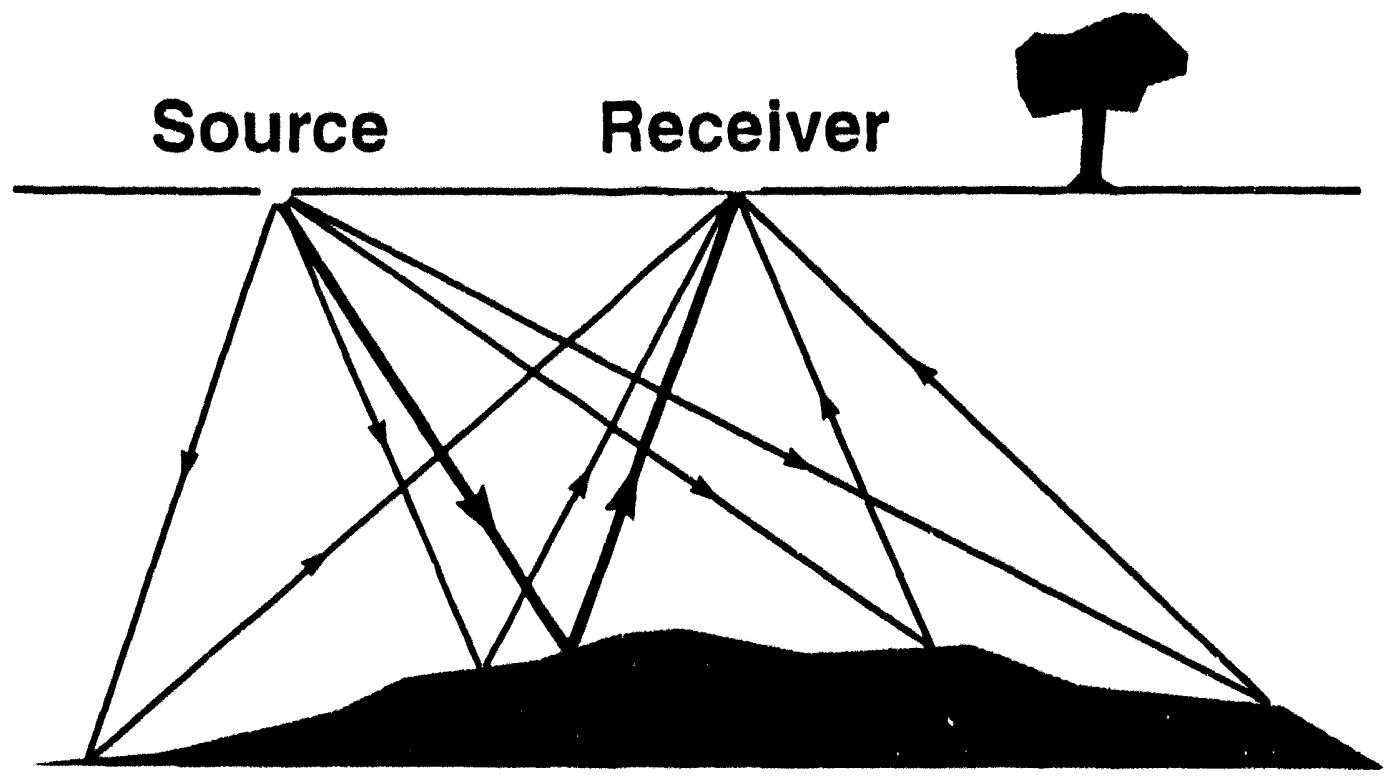

FIG. 2. 


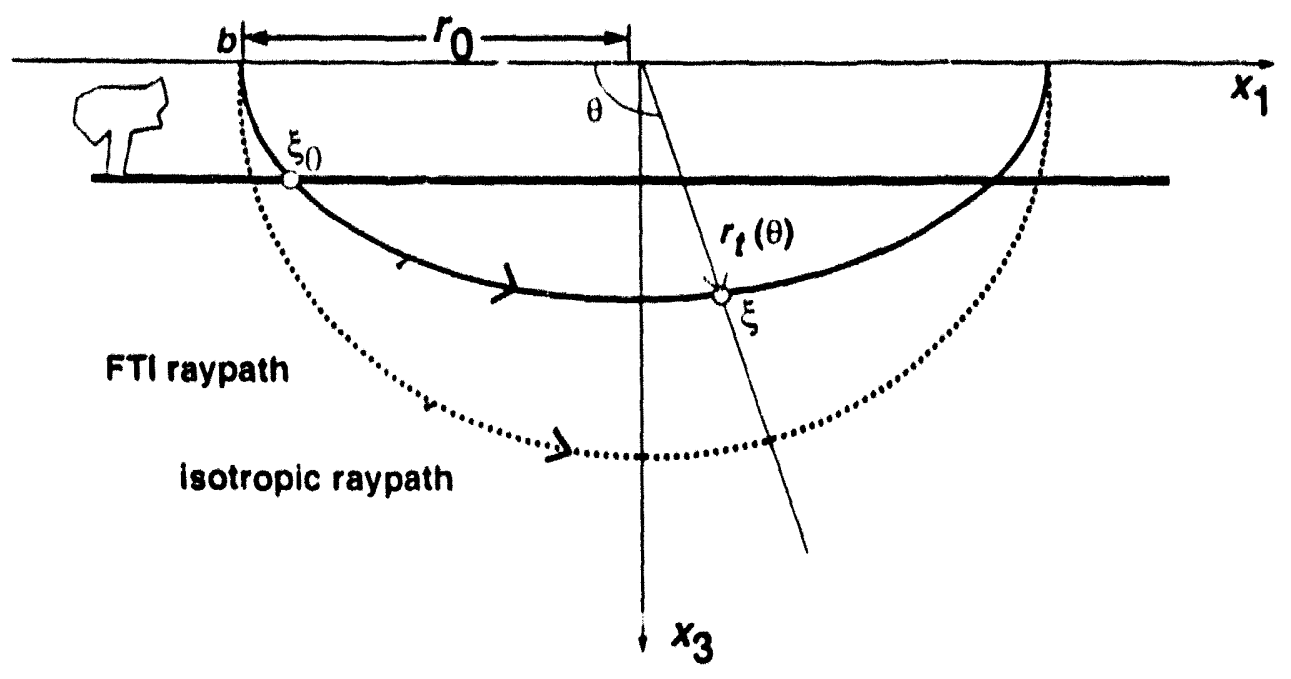

FiG. 3. 


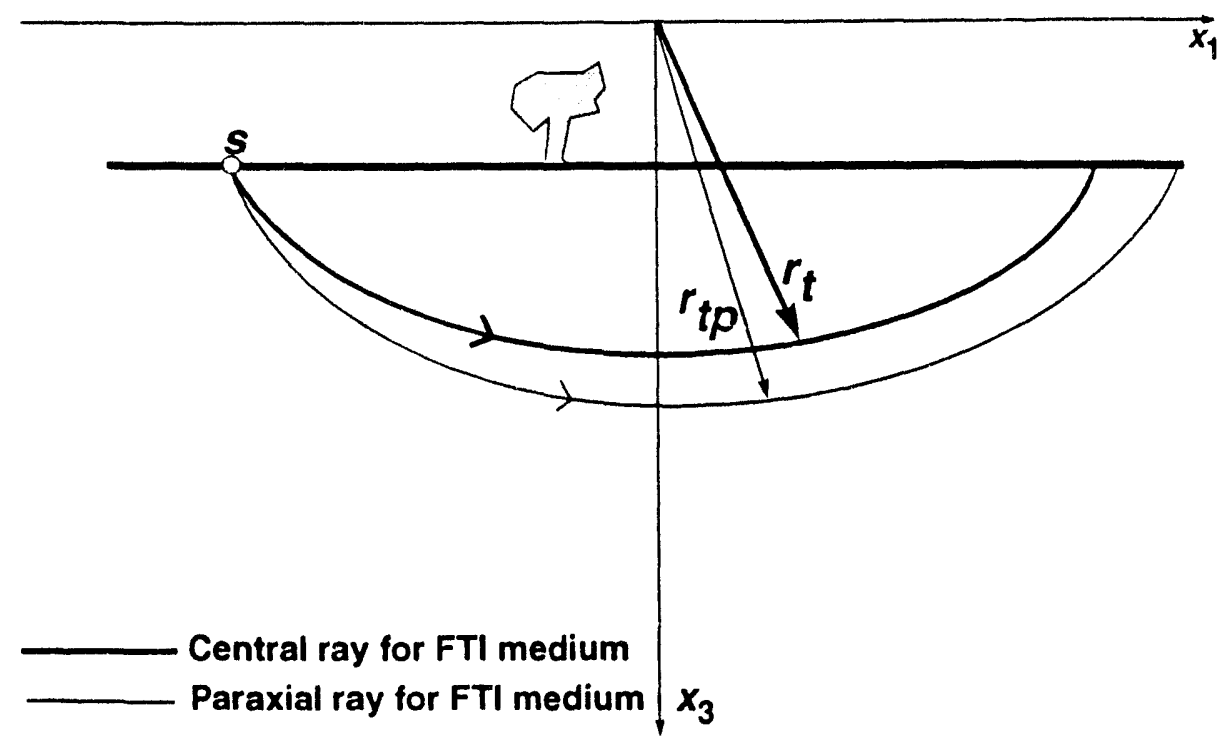

FIG. 4 . 



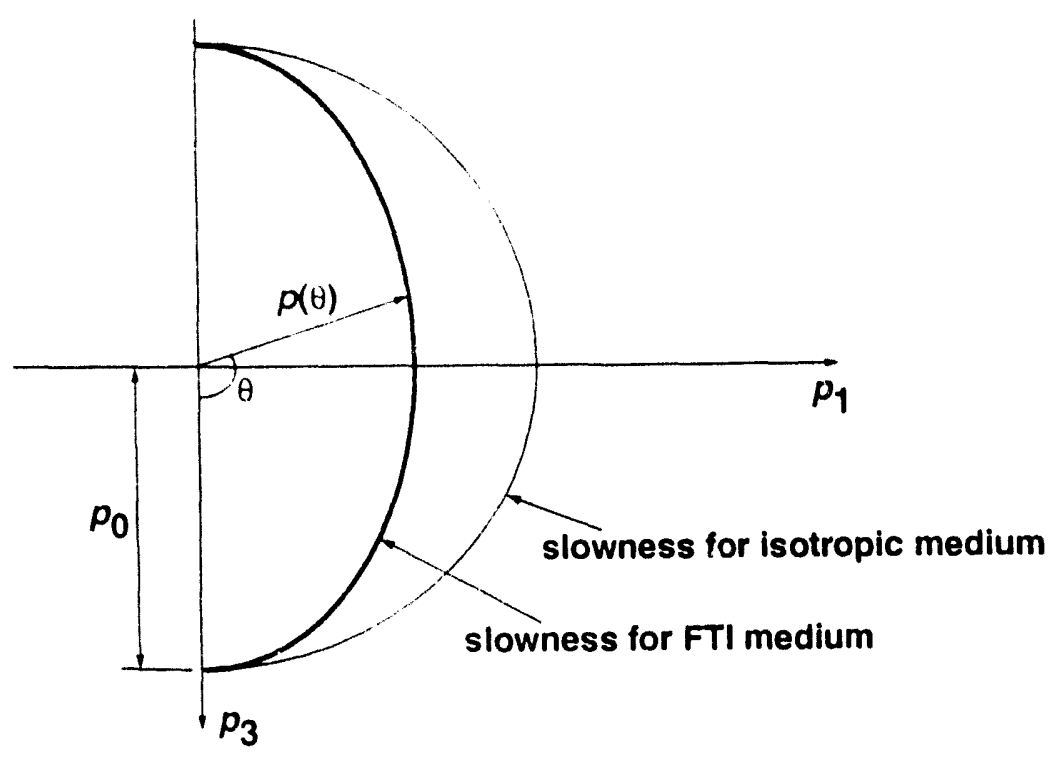

FIG. 6. 


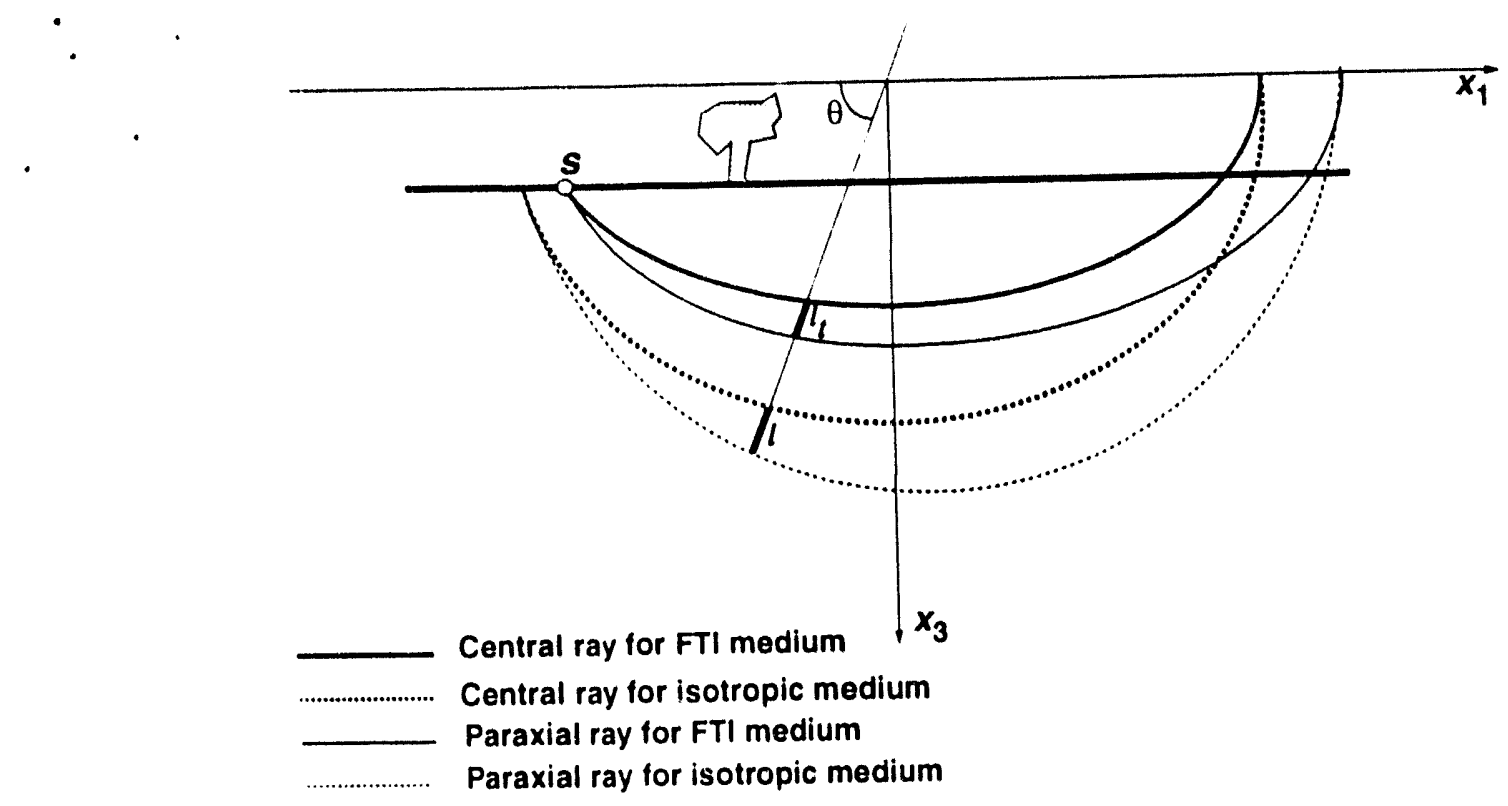

FIG. $\rceil.$ 


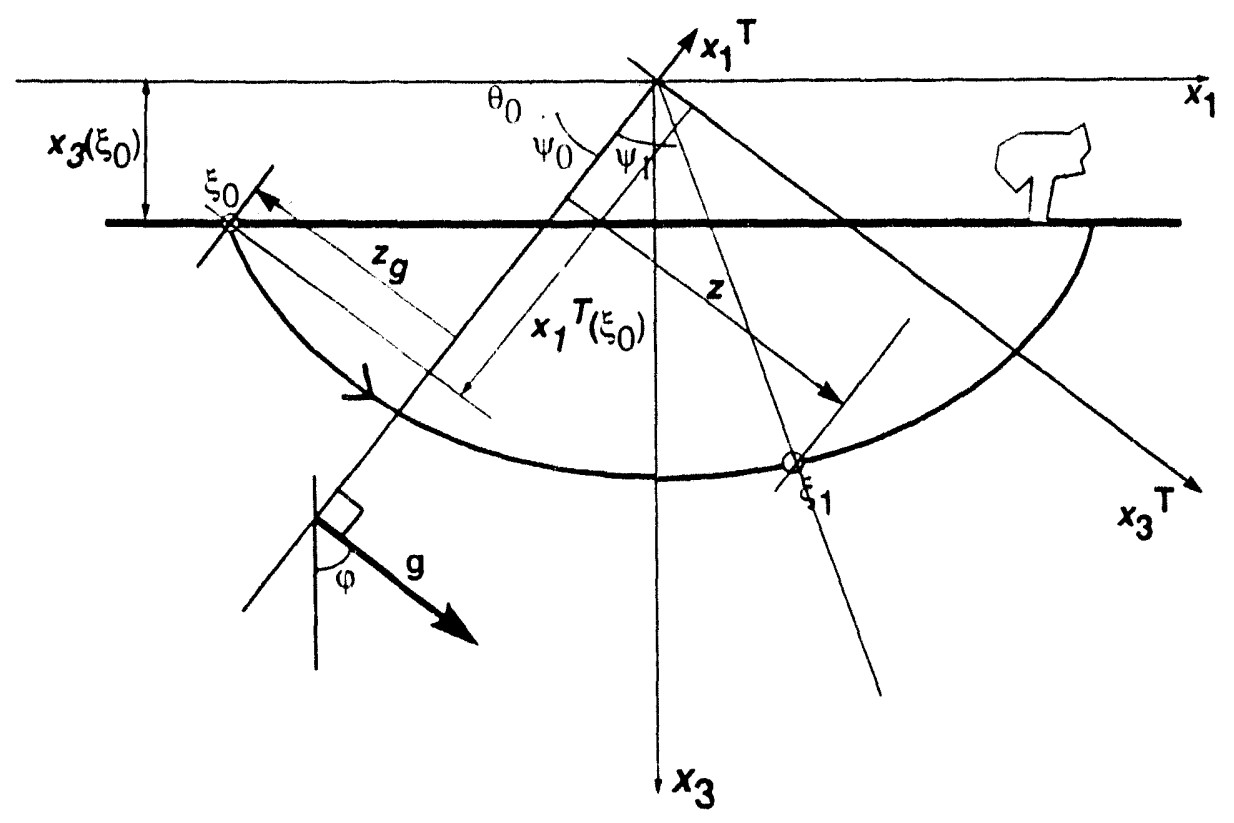

FIG. 8. 

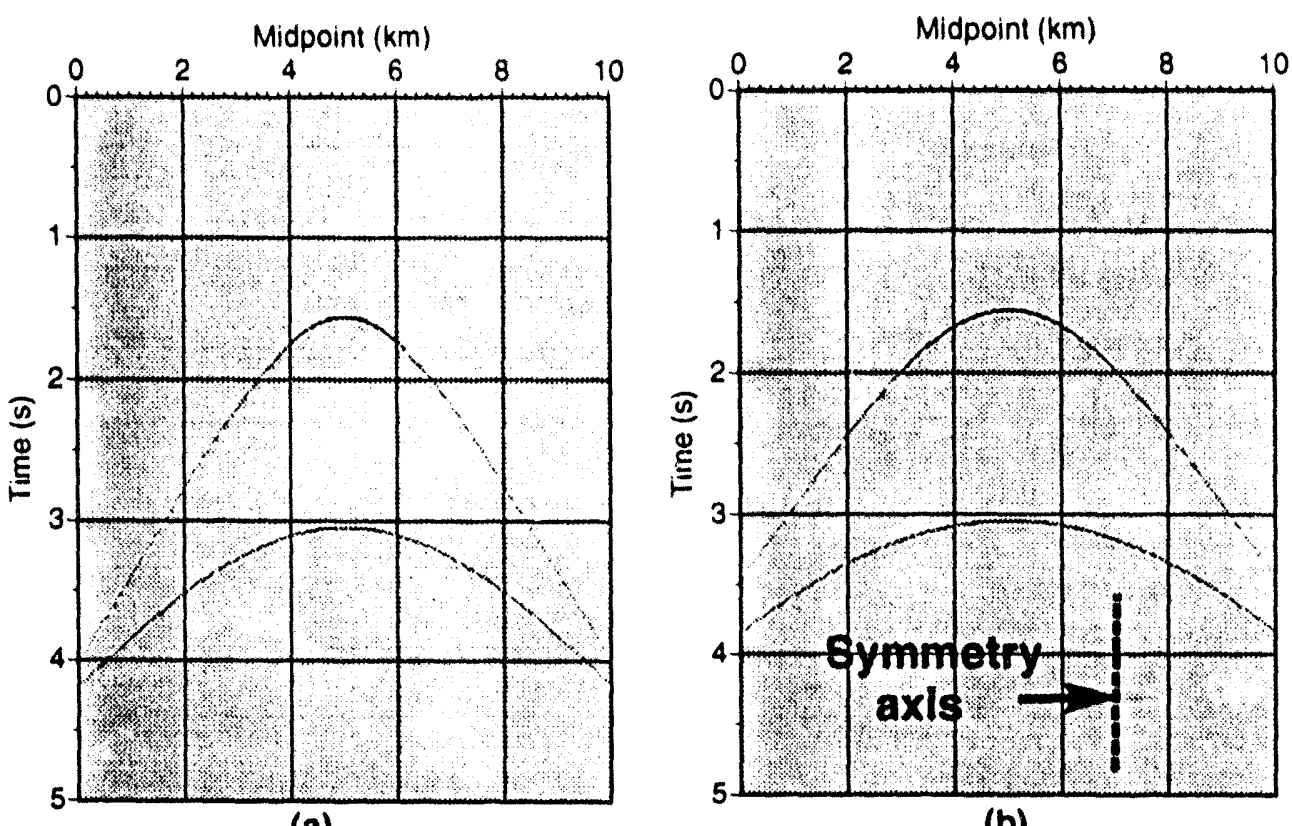

(b)

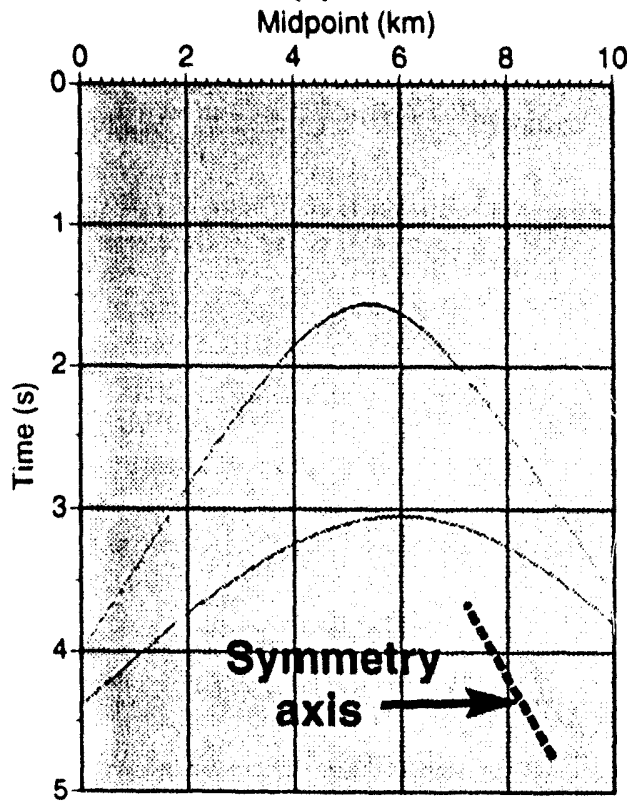

(c)

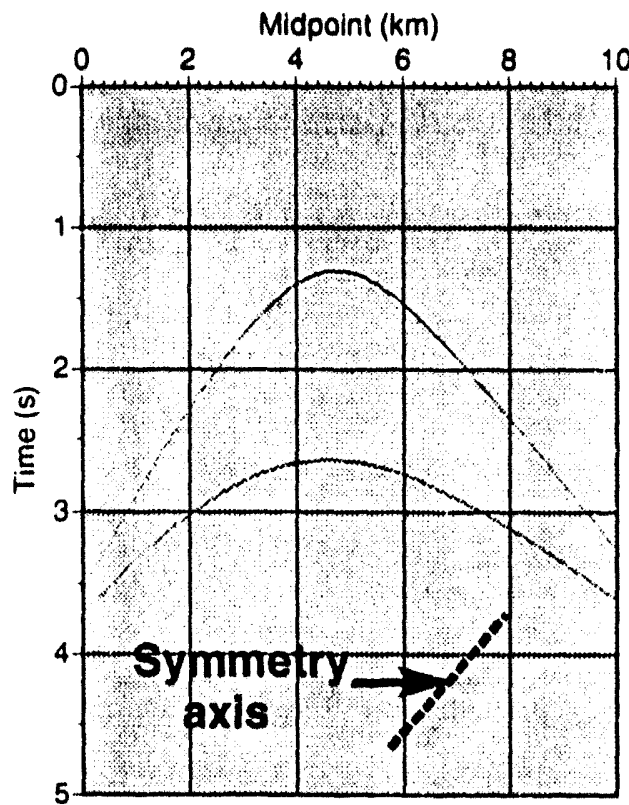

(d)

FiG. 9. 


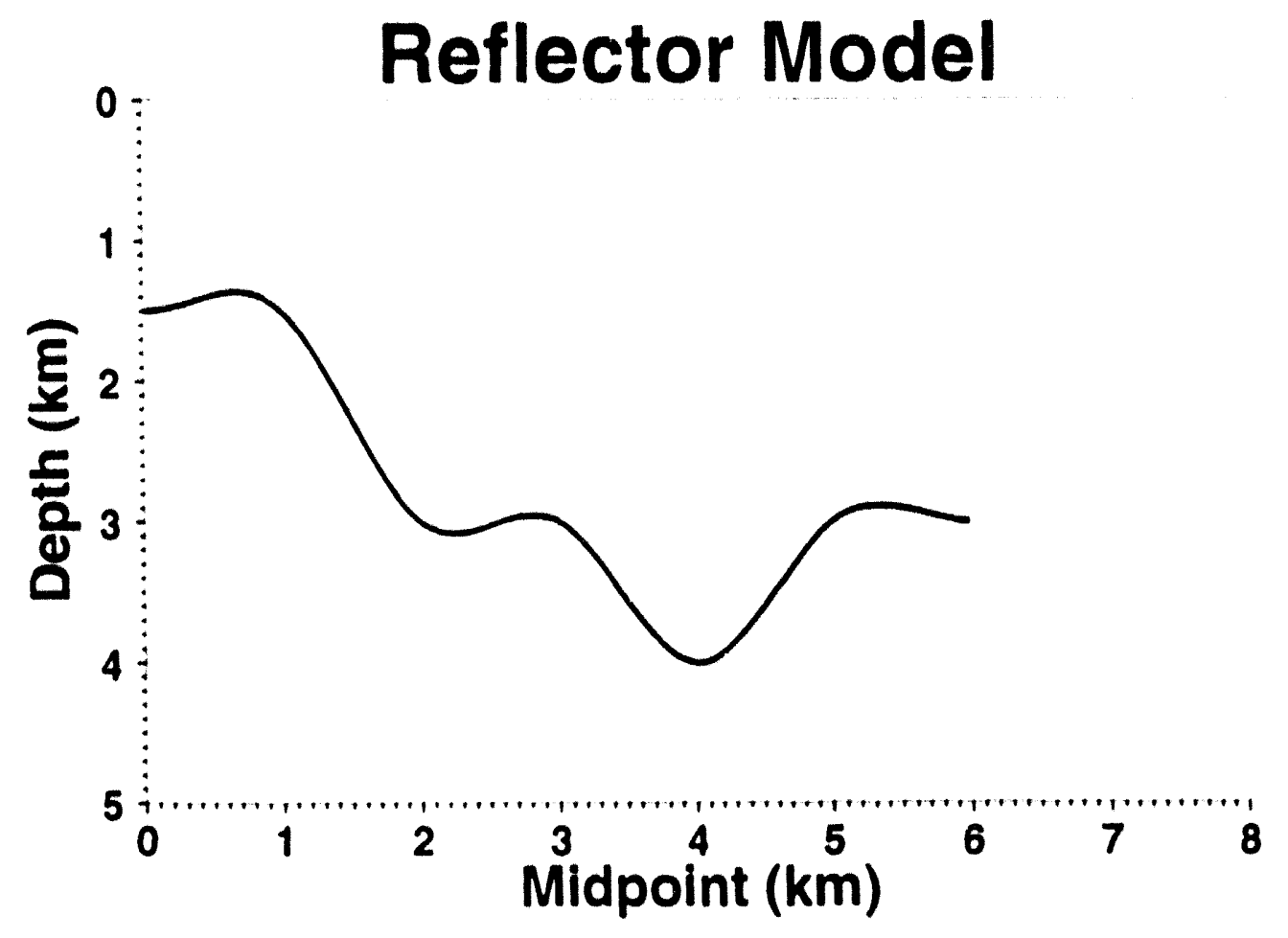

FIG. 10. 

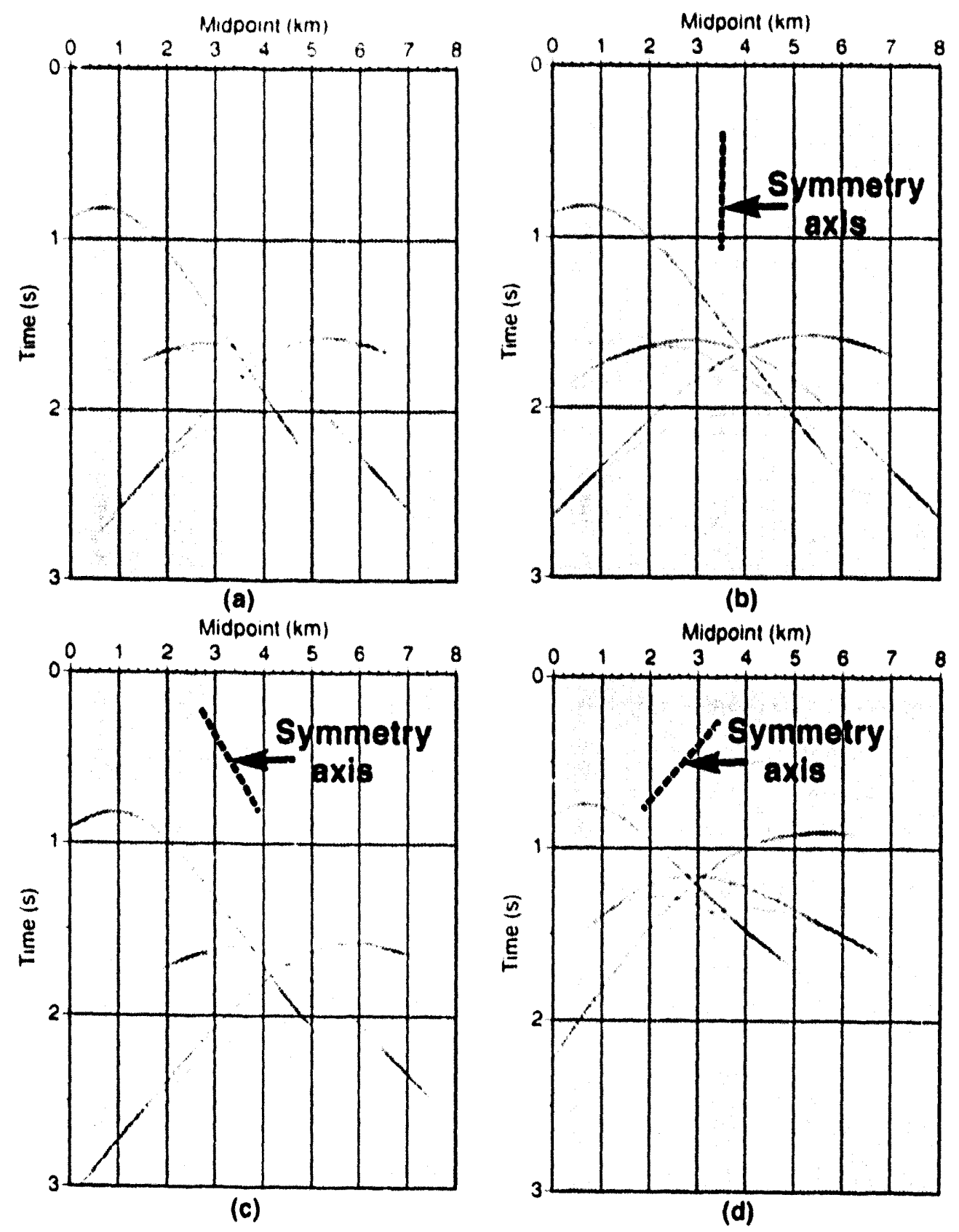

FIG. 11. 

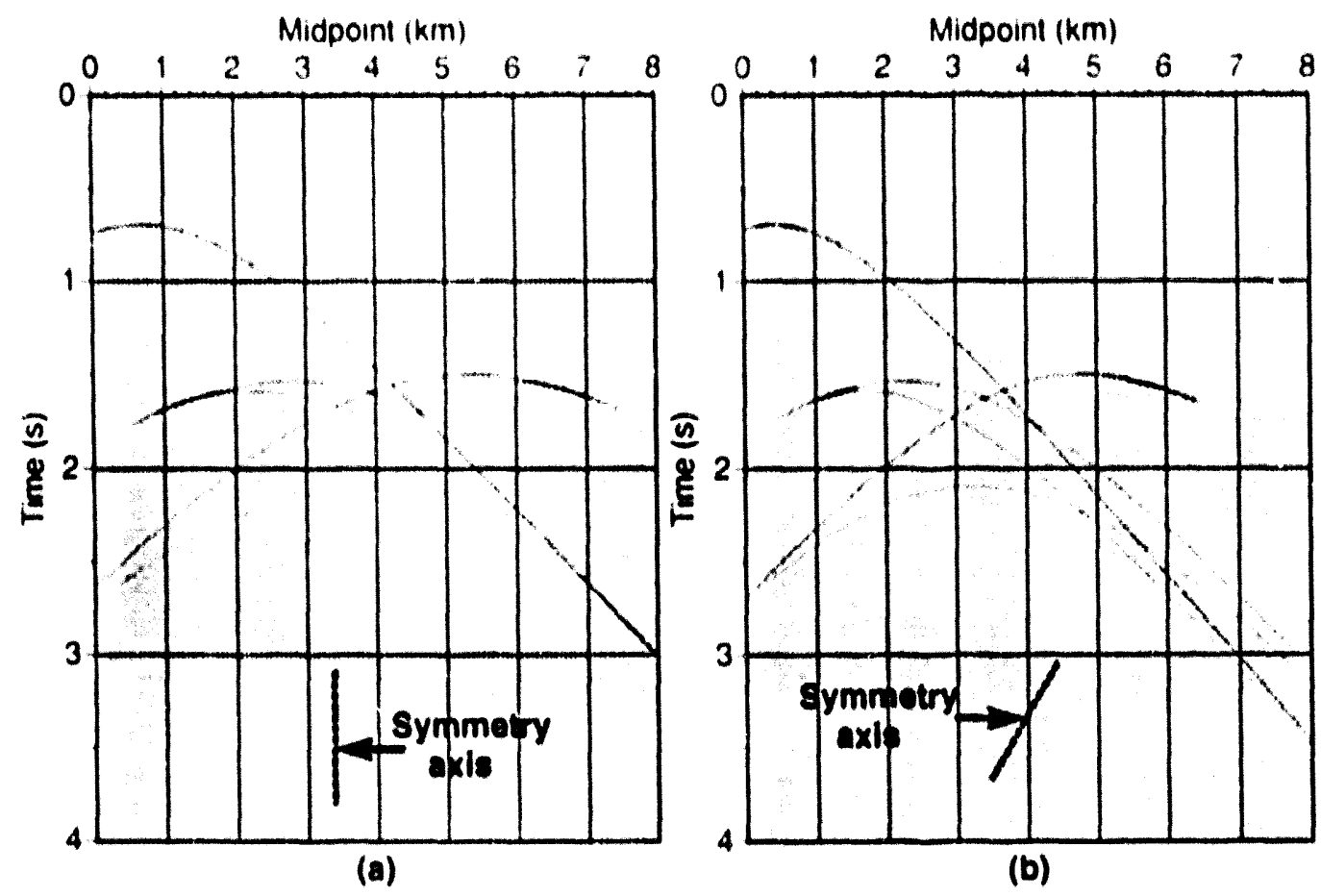

Fig. 12. 


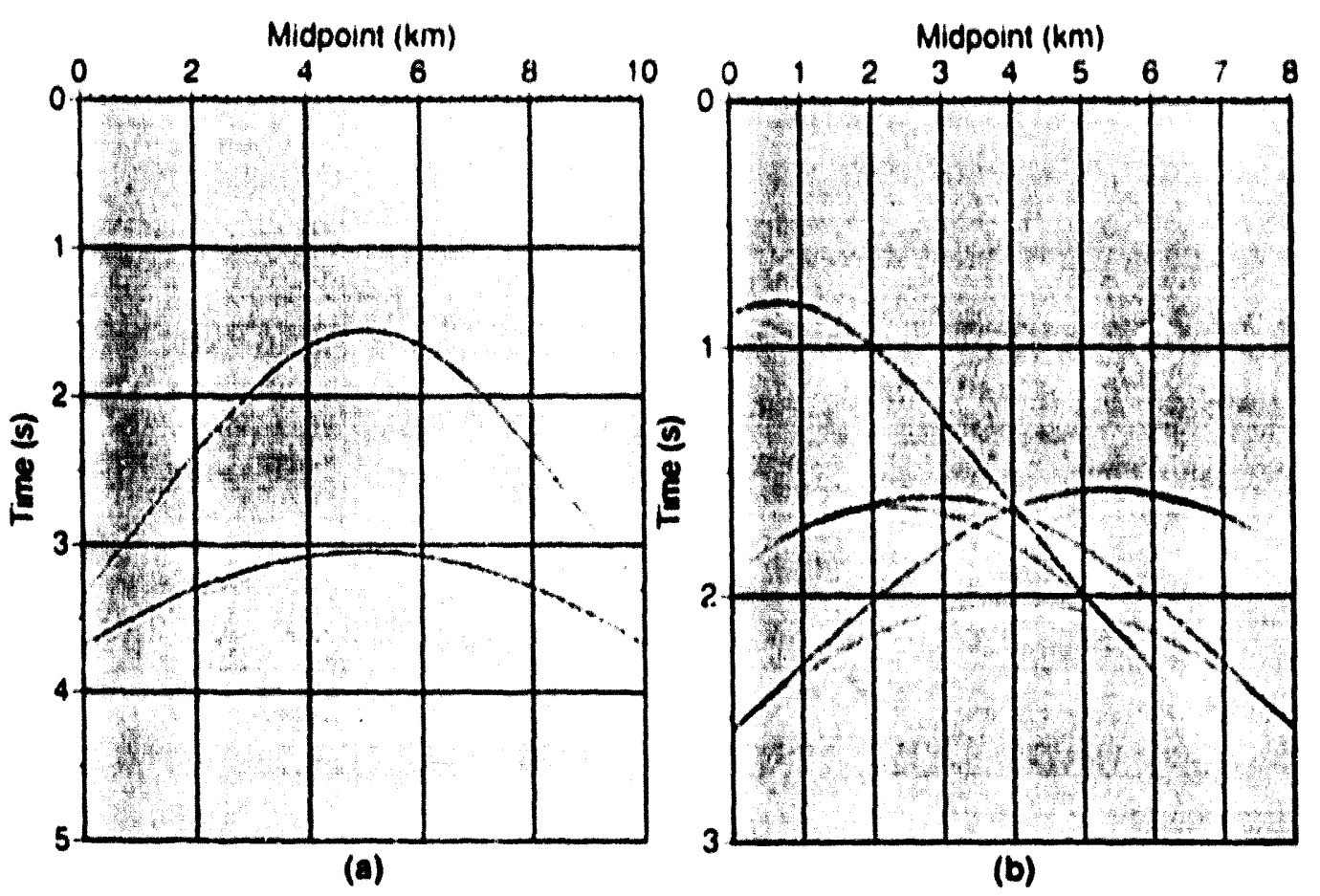

FIG. 13. 

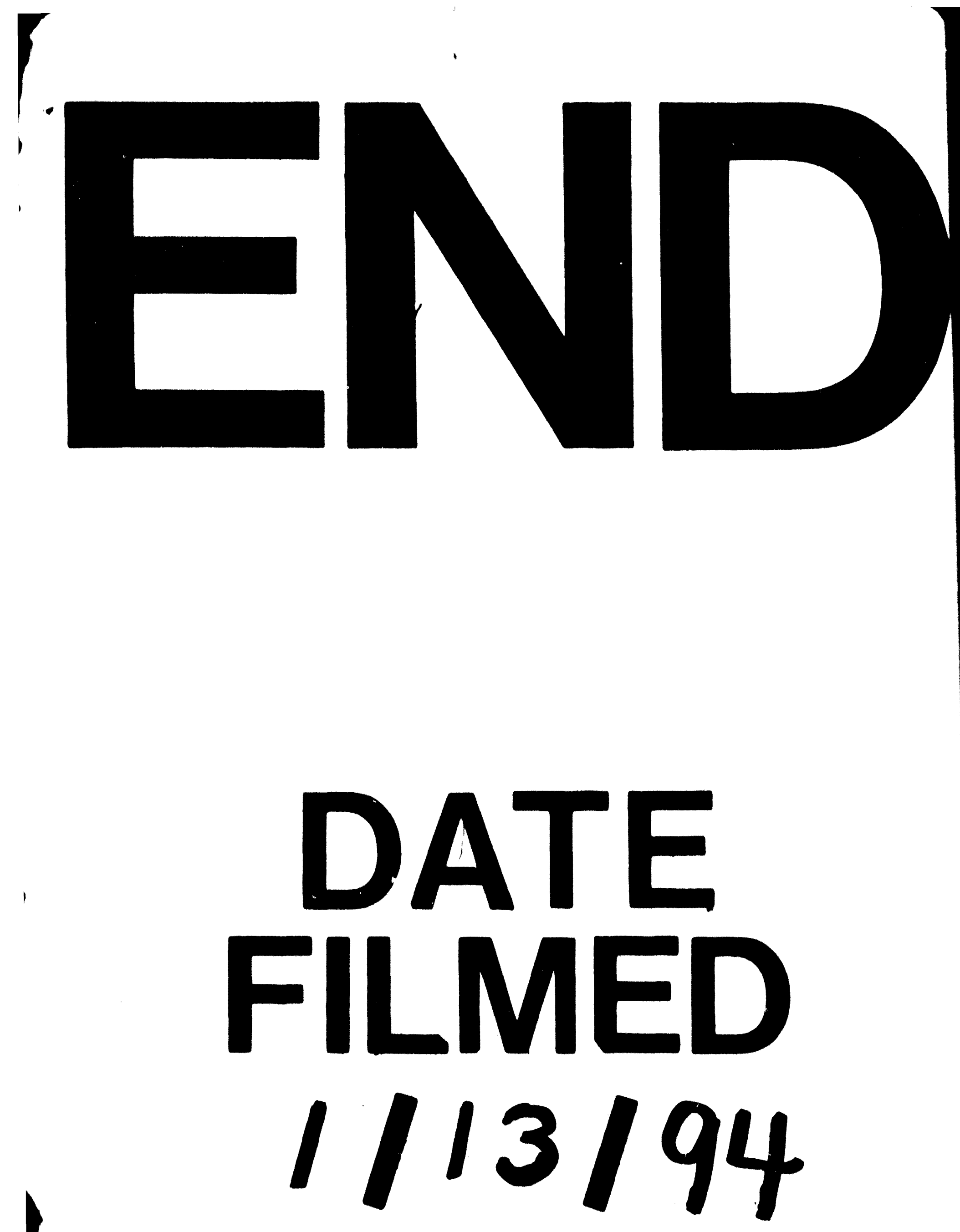

1 


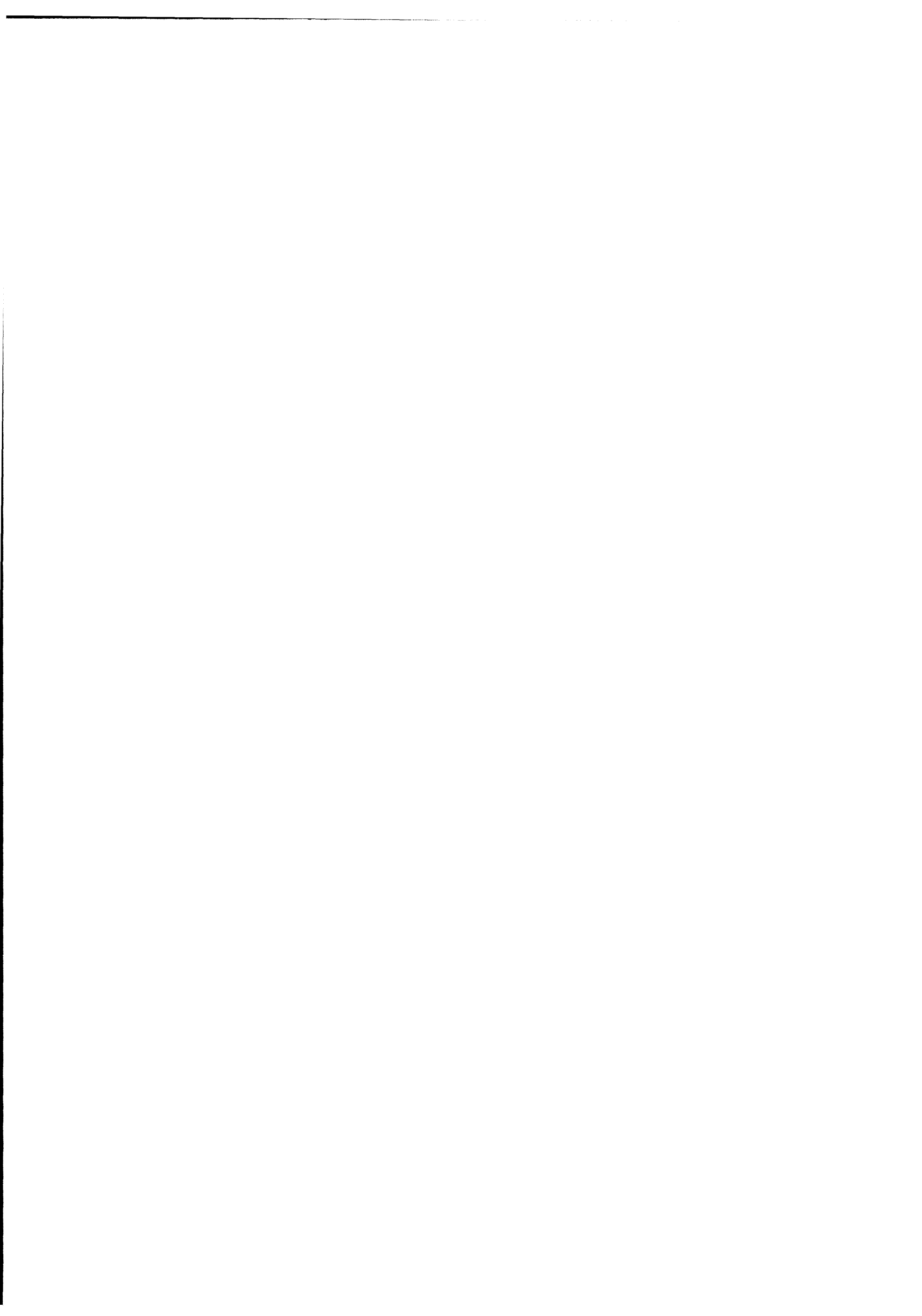

